\title{
Bibliographie de la Société de Notre-Dame de Montréal (1639-1663) accompagnée de notes historiques et critiques (suite)
}

\section{Marie-Claire Daveluy}

Volume 16, numéro 4, mars 1963

URI : https://id.erudit.org/iderudit/302246ar

DOI : https://doi.org/10.7202/302246ar

Aller au sommaire du numéro

Éditeur(s)

Institut d'histoire de l'Amérique française

ISSN

0035-2357 (imprimé)

1492-1383 (numérique)

Découvrir la revue

Citer ce document

Daveluy, M.-C. (1963). Bibliographie de la Société de Notre-Dame de Montréal (1639-1663) accompagnée de notes historiques et critiques (suite). Revue d'histoire de l'Amérique française, 16(4), 608-612.

https://doi.org/10.7202/302246ar 


\title{
BIBLIOGRAPHIE*
}

\author{
Bibliographie de la Société de Notre-Dame de Montréal \\ (1639-1663) \\ accompagnée de notes historiques et critiques
}

DEUXIÈME PARTIE

Bio-biliographie des Associés de Montréal

(suite)

Année 1648

45.-Ailleboust Des Musseaux (Charles d'), seigneur d'Argenteuil au Canada (1624-1700). Commandant du Camp volant créé en 1648; commandant intérimaire de VilleMarie (1651-1653) ; lieutenant de la garnison de Montréal (1654-1663); juge royal de Ville-Marie (1658 ?-1678).

\section{A. - NOTES BIOGRAPHIQUES}

Né en 1624, à Ancy-le-Franc, aux confins de la Champagne du sud-ouest, Charles d'Ailleboust était le fils de Nicolas et de Dorothée de Manthet, celle-ci fille de Jean de Manthet, sieur d'Argentenay et de Suzanne Hotman. Rappelons ici que Suzanne Hotman, l'aieule de Charles, fut la mère de Louis d'Ailleboust, le troisième gouverneur de la Nouvelle-France; car devenue veuve de Jean de Manthet, elle avait épousé Antoine d'Ailleboust, veuf lui aussi d'une première femme dont nous ignorons le nom, et dont il avait eu un fils, Nicolas, le père de Charles d'Ailleboust. "Nous sommes donc en présence, déclare Aegidius Fauteux, dans son ouvrage sur la Famille d'Ailleboust (Montréal, 1917), 30ss, d'un cas d'alliance compliqué. C'est après son second mariage avec Antoine d'Ailleboust que Suzanne

* Voir notre Revue, V: 139-147, 296-307, 445-460, 603-616; VI: 146150, 297-306, 458-463, 595-605; VII : 457-461, 586-592; VIII: 292-306, 449455 , 591-606; IX: 141-149, 306-309, 458-462, 594-602; X : 295-302 ; XI: $137-$ 142 , 298-304, 449-457, 608-614; XII : 144-147, 294-302, 443-453; XIII : $137-$ 149 , 298-305, 450-460, 594-602; XIV : $142-149,302-311,626-635$; XV : $141-$ 154, 466-472, 611-616; XVI: 294-307, 455-463. 
Hotman maria sa fille Dorothée, née de son premier mariage avec Jean de Manthet, à son beau-fils, Nicolas, né du premier mariage de son second mari. » Dorothée Manthet devenue Madame Nicolas d'Ailleboust se trouvait donc à la fois la demisœur, puis la belle-sœur de Louis d'Ailleboust, et son fils Charles était de ce fait doublement le neveu de notre gouverneur.

En 1648, âgé de 24 ans, Charles d'Ailleboust décida de s'établir au Canada «probablement sur les instances de son oncle Louis qui venait d'y être nommé gouverneur». Le jeune homme, un militaire de carrière, semble avoir possédé des connaissances juridiques. Elles motiveront plus tard sa nomination par le supérieur de Saint-Sulpice à Montréal, M. de Qeylus, vers 1658 ou 1659, au poste de juge royal de Ville-Marie, poste qu'il occupa durant vingt ans.

Oncle et neveu débarquèrent à Québec le 19 août 1648. Ils avaient fait la pénible traversée de l'Atlantique sur le navire le Cardinal. Ce séjour forcé, durant de longues semaines sur un vaisseau qui n'avançait qu'au gré de vents capricieux, leur permit de prendre des décisions sagement mûries concernant leur vie future dans la Nouvelle-France. La mort, en pleine mer, d'un personnage éminent du Canada, l'amiral Pierre Le Gardeur de Repentigny, dramatisa l'interminable voyage. La fin de l'amiral fut d'un chrétien admirablement résigné.

Dès l'automne de 1648, nous voyons Charles d'Ailleboust investi par le gouverneur, son oncle, du commandement du camp volant, de 40 hommes, nouvellement créé en vertu d'un édit daté du 5 mars 1648. Ce camp devait se porter au secours des colons canadiens menacés d'une attaque de l'armée iroquoise. Et cela en quelque lieu que ce fût dans la Nouvelle-France. "Ce corps militaire, remarque Aegidius Fauteux (ibid., 31) travailla si efficacement à la défense [surtout] de Montréal que deux ans plus tard on jugea à propos de porter son effectif à 70 hommes. 》 La réputation de bravoure et d'habileté stratégique dans la conduite de ses soldats grandirent aussitôt Charles d'Ailleboust dans l'estime de tous. Nous en voyons la preuve dans le geste de M. de Maisonneuve nommant à la veille de son départ pour la France en 1651, Charles d'Ailleboust, commandant intérimaire de Ville-Marie durant une absence qui allait durer deux ans. La situation critique des montréalistes, durant ces années de détresse, n'entama nullement le courage et l'activité de tous ces colons-militaires. Réfugiés au fort par ordre de M. de Maisonneuve, ils n'en continuèrent pas moins de vivre normalement. Il y eut des mariages, des baptêmes, quelques réjouissances, 
malgré des deuils inévitables. Charles d'Ailleboust donna luimême l'exemple du vouloir-vivre de chacun. La dernière semaine d'août 1652 il quittait en barque Ville-Marie qu'il avait mise en solide état de défense. Le 4 septembre 1652, devant Guillaume Audouard, notaire royal à Québec, il signait son contrat de mariage avec Catherine Le Gardeur de Repentigny, fille de l'amiral Pierre Le Gardeur de Repentigny dont nous avons parlé du décès en mer quatre ans plus tôt, et de Marie Favery, une femme d'élite dont l'intendant Talon devait, une dizaine d'années plus tard, faire un magnifique éloge. Catherine, la brave petite fiancée, née en France en 1634, comptait dix-huit ans. La perspective d'aller habiter Ville-Marie-la-Sanglante, ne l'effraya nullement. Les jeunes filles de l'époque, tout comme leurs frères, faisaient preuve d'une humeur héroïque vraiment admirable.

Charles d'Ailleboust des Musseaux fut le premier du nom à faire souche au Canada. Sa femme lui donna quatorze enfants. Mais ici, il faut citer l'appréciation d'Aegidius Fauteux sur cette famille appartenant à la vieille noblesse française. «La famille d'Ailleboust, écrit ce maître généalogiste, occupe dans nos annales une place aussi large qu'honorable. Quoiqu'éteinte aujourd'hui, elle reste sans contredit une des plus importantes du Canada français. Son association avec cette colonie date des premiers temps. Elle a donné à la Nouvelle-France un gouverneur et quelques-uns de ses guerriers les plus glorieux. Peu de familles canadiennes peuvent se vanter d'ailleurs d'avoir une origine aussi ancienne. Avant même de s'établir au Canada, la famille d'Ailleboust s'était assurée en France un rang distingué; et dès les commencements du XVIe siècle, plusieurs de ses membres y occupaient déjà de hautes situations.» ${ }^{45}$

45 Sans doute, comme l'écrit Aegidius Fauteux, op. cit. [7], la famille d'Ailleboust est aujourd'hui éteinte. Mais à travers les générations des descendants de Charles d'Ailleboust, et à cause des alliances contractées au cours de trois siècles, nous connaissons encore aujourd'hui des personnes éminentes comptant des aïeux et des aïeules ayant porté le nom de d'Ailleboust. Nous avons l'honneur de citer comme exemple de notre assertion, Son Excellence Monseigneur Joseph-Conrad [Guillet de] Chaumont, dont le premier ancêtre canadien, Nicolas-Auguste Guillet de Chaumont, notaire royal et écrivain du roi, d'origine parisienne, était venu s'établir au Canada vers 1720 , à l'âge de 24 ans. Il épousait, en secondes noces, à Montréal, le 17 juin 1737, Félicité-Josèphe d'Ailleboust des Musseaux (1706-1772). Leur fils cadet, Joseph, né en 1739, devint plus tard juge de paix et co-seigneur de Boucherville. Il s'unissait à Terrebonne, le 15 avril 1765 , à sa cousine germaine, Marie-Catherine, fille de Pierre-Joseph d'Ailleboust de Manthet des Musseaux (4e génération d'un des fils de Charles d'Ailleboust et de Marie-Jeanne de Goutins). Madame Joseph Guillet de Chaumont (1746-1796) est la trisaïeule de Mgr [Guillet de] Chaumont, évêque d'Arena et auxiliaire de Montréal. Nous avons plaisir à ajouter que, lors des fêtes 
Jusqu'en 1663 M. des Musseaux agit à Ville-Marie en qualité de lieutenant de la garnison de Montréal. En 1664 un malheureux événement, un véritable abus d'autorité de la part du gouverneur de la Nouvelle-France, M. de Mésy, vint modifier les choses à Ville-Marie. "Sans avoir égard aux prérogatives des Messieurs de Saint-Sulpice qui venaient d'acquérir tous les droits de la Société fondatrice de Montréal, à titre de nouveaux seigneurs, et qui pouvaient par conséquent exercer haute et basse justice à Ville-Marie, M. de Mésy créait pour toute l'île de Montréal, une sénéchaussée royale avec M. de Sailly comme juge. La Compagnie de Saint-Sulpice s'y opposa avec raison et mit aussitôt en fonction une autre sénéchaussée dont Charles d'Ailleboust des Musseaux devint le juge. Il fallut trois ans de protestations avant que Saint-Sulpice parvint à faire cesser ce désordre de deux sénéchaussées agissant concurremment. 》M. Fauteux doute cependant que M. d'Ailleboust ait été investi de cette charge en cette mémorable occasion. Il cite une pièce judiciaire produite devant le Conseil souverain au mois d'août 1677 et où il est démontré que Charles d'Ailleboust des Musseaux exerçait les fonctions de juge civil et criminel à Montréal depuis 18 ans, et qu'il en avait été alors pourvu par le supérieur de Saint-Sulpice, M. de Queylus. "Sa première commission [en ce cas], explique M. Fauteux, émanerait de la Société de Notre-Dame de Montréal vers 1659 , peut-être en 1658 , pendant le voyage que Charles d'Ailleboust entreprit en France avec son oncle Louis et M. de Maisonneuve afin de conférer avec M. Olier dans l'intérêt de Ville-Marie. ${ }^{46}$

En 1677 les Messieurs de Saint-Sulpice nommèrent comme baillif et juge civil et criminel à Montréal, Jean-Baptiste Migeon de Branssac, licencié ès-loi et advocat [sic] au Parlement de Paris. Ils révoquaient, par le fait même, M. d'Ailleboust des Musseaux qui protesta mais inutilement. Le Conseil, par un

religieuses et civiles commémorant le troisième centenaire de la fondation de Montréal (1642-1942), Mgr [Guillet de] Chaumont - et ce fut là un bien juste retour des choses - était nommé président général des fêtes religieuses. Avec lui se trouvait de nouveau à l'honneur le beau nom des d'Ailleboust de Manthet et des Musseaux porté par ses aïeules de la troisième et quatrième génération de cette famille glorieuse. Mgr Chaumont, comme beaucoup de nos anciens nobles, laissa tomber, comme ses père et grand-père, le nom patronymique de sa famille et la particule nobiliaire.

46 Fauteux, op. cit., 31. M. Fauteux fait erreur en fixant le voyage de MM. d'Ailleboust et Maisonneuve à la date de 1658 ou 1659. Ce voyage eut lieu en 1655. Par ailleurs, M. Olier, en 1658, était décédé depuis un an, en avril 1657. (Voir Faillon, Histoire de la colonie française, II : 268; aussi le Journal des Jésuites (1871, éd. Laverdière et Casgrain), 217 et 218). 
arrêt du 20 septembre 1677, « confirma la nomination de Migeon de Branssac, les ecclésiastiques de St-Sulpice ayant le droit de nommer les juges durant bon plaisir dans les limites de leurs seigneuries ». Chose curieuse aucun motif n'avait été donné lors de cette mutation dans la magistrature montréalaise. M. Migeon de Branssac avait épousé, plusieurs années avant sa nomination, la nièce de M. Gabriel Souart, sulpicien, Catherine Gaucher de Belleville, venue de France avec Jeanne Mance, en 1659. M. des Musseaux acquérait, en 1680, un fief connu sous le nom d'Argenteuil. Il obtenait de l'intendant Duchesneau cette concession située au-dessus de l'île de Montréal. Le gouverneur Frontenac, en 1682, ratifia la possession de ce fief. M. des Musseaux le vendit alors à son fils Pierre, mais cette transaction ne fut définitivement résolue qu'en 1725 par l'intendant Bégon.

Charles d'Ailleboust des Musseaux mourut à Montréal en 1700. Son acte d'inhumation est rédigé comme suit dans les registres de Montréal: «Le vingtième jour de novembre mil sept cent, sépulture de Charles d'Ailleboust, un des Messieurs de l'ancienne Compagnie [de Montréal], mort le 19, à l'âge de 76 ans. » Ce texte confirme de façon formelle son titre d'associé de Montréal. Il est évident qu'en 1648, avant de quitter la France, son oncle, Louis d'Ailleboust de Coulonge, le présentait aux Messieurs et Dames de la Société de Montréal comme une importante recrue personnelle. Le troisième gouverneur de la Nouvelle-France fut sans cesse, du reste, en relations avec les associés-fondateurs et l'un des rares, parmi ceux-ci, à bien connaître et à traiter, - extraordinaire privilège - en compagnie de Messieurs de La Dauversière et Fancamp, des transactions importantes avec Mme de Bullion, «la bienfaitrice inconnue du Montréal ». Remarquons aussi la prédilection qu'il manifesta pour le fils de sa demi-sœur, Dorothée de Manthet. Il l'aida de toutes façons à bien s'établir au Canada, à y poursuivre sa carrière militaire; puis, plus tard, il contribua à sa nomination par les Messieurs de Montréal au poste élevé de juge royal de Ville-Marie. Au sujet de la succession de son oncle dont il fut l'un des héritiers, Charles d'Ailleboust eut quelques démêlés avec sa veuve, la pieuse Barbe de Boullongne, mais tout finit par s'arranger à l'amiable. Le premier d'Ailleboust à faire souche au Canada fut vraiment un officier de mérite et d'un beau courage, un juge intègre à la tâche durant vingt ans; et enfin un chef de famille dont les nombreux enfants héritèrent de sa droiture de cour et d'esprit et de l'attachement profond qui le liait à sa nouvelle patrie, le Canada.

(à suivre)

MARIE-ClaIRE DAVELUY, de l'Académie française. 